\title{
MODELING OF A NANOCYLINDER
}

\author{
Serghei A. Baranov ${ }^{1,2}$ \\ ${ }^{I}$ Institute of Applied Physics, str. Academiei 5, Chisinau, MD-2028 Republic of Moldova \\ ${ }^{2}$ Shevchenko Pridnestrov'e State University, str. 25 Oktyabrya 128, Tiraspol, \\ Republic of Moldova \\ E-mail: baranov@phys.asm.md
}

(Received October 15, 2020)

https://doi.org/10.53081/mjps.2021.20-1.03

CZU:538.9+532.511

\begin{abstract}
The results of the theory of modeling for obtaining nanocylinders have been described. A case of a nanocylinder whose diameters are shorter than the Tolman length has been considered. This important issue is taken into account in studying a nanocylinder for which, in the simplest model, the thickness of the interfacial layer cannot be determined because it supposedly has a small size. At the same time, it has been shown that the introduction of a special form of anisotropy energy makes it possible to analytically describe the origin of an interfacial layer whose sizes can be regarded as sizes comparable to the Tolman length.
\end{abstract}

Keywords: nanocylinder, Tolman length, Heisenberg model, Euler-Lagrange equations, instantons or skyrmions

\section{Rezumat}

Sunt descrise rezultatele teoriei modelării pentru obținerea nanocilindrilor. Am considerat un caz în care nanocilindrul are diametre mai mici decât lungimea Tolman. Această întrebare importantă este luată în considerare atunci când se studiază nanocilindrul pentru care în cel mai simplu model nu s-a putut distinge grosimea stratului interfacial datorită dimensiunii sale presupuse mici. În același timp, se arată că introducerea unei forme speciale de energie anizotropică permite o descriere analitică a originii unui strat interfacial ale cărui dimensiuni pot fi considerate ca dimensiuni comparabile cu lungimea Tolman.

Cuvinte cheie: nanocilindru, lungime Tolman, model Heisenberg, ecuații EulerLagrange, instantane sau skyrmions

\section{Introduction}

The modern statistical mechanics of curved interfaces, mainly spherical and cylindrical, is equally important to the simple thermodynamics of planar interfaces (maybe even more important). However, this statistical mechanics theory needs a more subtle analysis than the 
thermodynamics of flat geometry, for which a great progress has been achieved in understanding the properties (for details see [1-5]).

The first studies of the effect of only one curvature on the properties of drops (Jung and Laplace) date back to the nineteenth century [1,2]. Laplace considered a drop of radius $R$ enclosing a homogeneous liquid-like phase (interior phase) separated from a homogeneous bulk vapor phase (exterior phase) by a mathematical dividing surface, where the density changes abruptly from a constant value inside the drop to a constant value outside; to provide the stability of the drop against the surface tension (regarded as a mechanical force) of the vapor-liquid interface, it is necessary to set up a pressure difference over the interface to balance the contracting force and maintain the system in equilibrium, i.e., the condition of mechanical equilibrium, which is referred to as the Laplace law (see [1-10]).

On the other hand, the curved interface theory with a Tolman's intermediate phase was based on sound thermodynamic arguments in the late 1940s (see [6]); however, it received little attention in electrochemistry (see, for example, [3-5]). After that, this theory was developed in [7-10]. We will not discuss all reviews; we can only mention many original papers and reviews [11-20]. Those studies are close to our research.

Below, we will consider a new theory [21-24] that takes into account the cylindrical shape and the intermediate phase. Moreover, the size of the intermediate phase can be arbitrary.

\section{A Small Long Cylinder}

We consider a case of the application theory [21-23], where nanoparticles have the form of a long cylinder. We used a cylindrical coordinate system for which the characteristic spin function $[21,22]$ is represented by angle function $\theta(r)$ about the cylinder axis $z$.

The free energy in this model can have the following form [21, 22]:

$$
H_{g, c}=\frac{A}{2}\left[\theta^{\prime 2}+\frac{\sin ^{2} \theta}{r^{2}}\right]+k_{1} \frac{\sin ^{4} \theta}{r^{2}}+\ldots
$$

where $\theta(r)$ is the angle between the cylinder axis and the magnetization vector; $r$ is the radial coordinate, and $k_{1}$ is the second anisotropy constant.

The model kinetic energy in (1) is a classical analog of the exchange energy in the Heisenberg model for the two-dimensional space at the continuum approximation [21], which in our case corresponds to the infinite cylinder model [21, 22]. In this case, the kinetic energy in (1) coincides in form with the kinetic energy of the particle (in cylindrical coordinates). This fact is not casual because the model under consideration permits exact analytical solutions in the form of quasi-particles (nonlinear waves), which are referred to as instantons (or skyrmions [21, 22]). Note that, in our case, these quasi-particles are topological compositions, rather than dynamic particles. Therefore, in our case, the virtual kinetic energy of a topological instanton is meant by the kinetic energy.

We introduce a relative coordinate

$$
\rho=\frac{r}{R_{c}}
$$


where $R_{c}$ is the drop equilibrium radius.

In this case, there is a condition of $0 \leq \rho \leq 1$. The proposed continuum model of energy (1) actually appears to be a Heisenberg model, in which the interacting spins have the meaning of energy states of the particles associated with the constant exchange interaction $A$ (with the dimension for the exchange energy $[\mathrm{J} / \mathrm{m}])$.

Using (1), it is simple to derive the Euler-Lagrange equation:

$$
\theta^{\prime \prime}(\rho)+\frac{\theta^{\prime}(\rho)}{\rho}-\frac{\sin \theta \cos \theta}{\rho^{2}}-k_{1} \frac{\sin ^{3} \theta \cos \theta}{\rho^{2}}-\ldots=0
$$

For simplicity, it is sufficient to use only a particular solution of this equation describing the nucleation process under simple boundary conditions:

$$
\theta(\rho)= \begin{cases}\pi, & \rho=0, \\ \frac{\pi}{2}, & \rho=1 .\end{cases}
$$

The solutions of equations (3) and (4) are as follows:

$$
\begin{aligned}
& \tan \left(\frac{\theta}{2}\right) \sim \frac{1}{\rho}\left[1-k_{1} \rho^{2}-\ldots\right], \\
& k_{1}<1 \\
& \text { (if } \left.\quad k_{1}=0, \text { then } \quad \tan \left(\frac{\theta}{2}\right)=\frac{1}{\rho}\right),
\end{aligned}
$$

which is convenient for further analysis.

Let us introduce the model surface energy to obtain the Euler-Lagrange equations for the scale-invariant theory as well:

$$
\theta_{a}^{\prime \prime}(\rho)+\frac{\theta_{a}^{\prime}(\rho)}{\rho}-\frac{a^{2} \sin \theta_{a} \cos \theta_{a}}{\rho^{2}}=0,
$$

where $a^{2}$ is the ratio of the anisotropy energy to exchange interaction constant $A$. The $a^{2}$ parameter is determined in [21,22]:

$$
a^{2}=\frac{B}{A}+1
$$

with the determined anisotropy function (model as the Rapini modified potential (see [21, 22]):

$$
\frac{B \sin ^{2} \theta_{a}}{2 \rho^{2}}
$$

where $B$ is the positive energy quantity whose dimension coincides with $A$.

For agreement with the previous solution, we assume that, in (6), anisotropy does not occur at $B=0$ and occurs at $B>0$. The solution of equation (6) is as follows: 


$$
\tan \left(\frac{\theta_{a}}{2}\right)=\frac{1}{\rho^{a}} .
$$

Note that solutions (5) and (9) analytically join; therefore, the indices are later omitted.

Let us consider one general solution (9). The diagram of this solution is shown in Figs. 1a and $1 b$.

It is easy to show that the $\theta(r)$ function has no point of inflection at $a=1$ and $0 \leq \rho \leq 1$. This point appears only at $a>1$. This means that the surface layer in our model can exist only at $a>1$. In this case, a certain volume whose energy is the surface energy of the cylindrical particle can be chosen as a surface layer. For definiteness, we suppose, for example, that the surface layer begins to clearly manifest itself from a value of $a>4$. Thus, we suppose that at $a=1$ there is no anisotropy in the system, and the Tolman length actually coincides with the drop sizes. If $a>1$, then, in the proposed model, the specific anisotropy is higher than the exchange interaction, and in the drop there appears a parameter (Tolman length) that characterizes the dimension of the interfacial region.

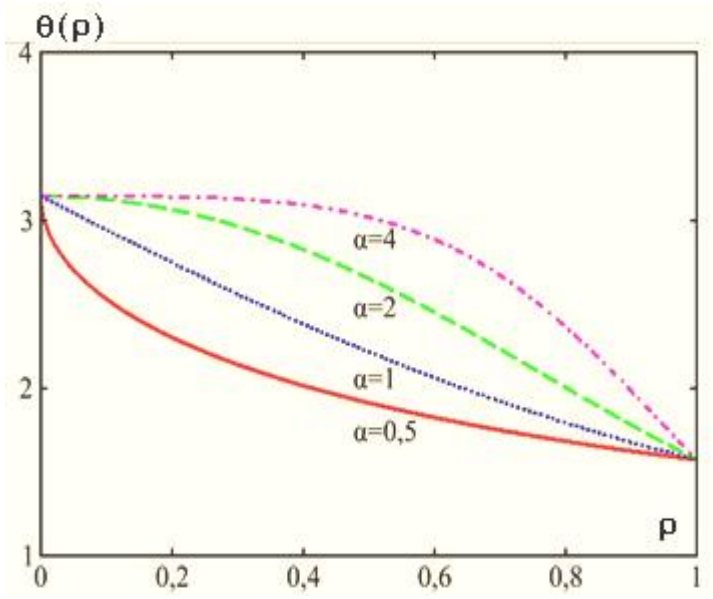

Fig. 1a. Diagrams of solution (9) at different values of parameter $a$ (see [22]).

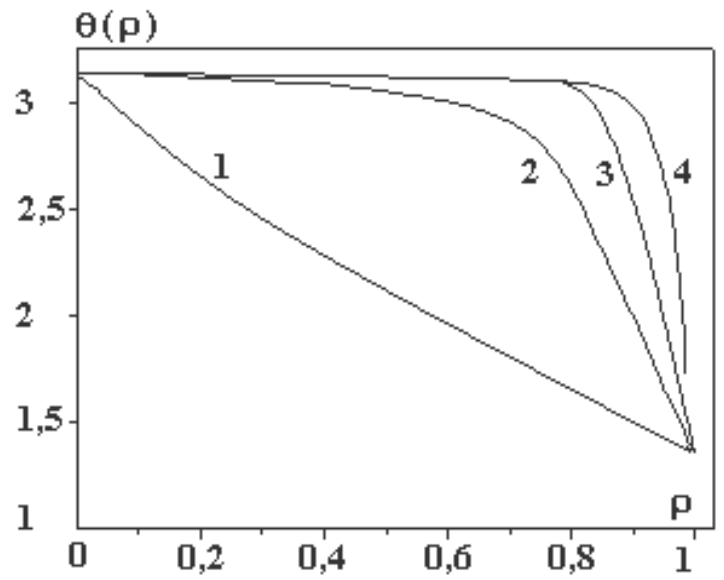

Fig. 1b. Diagrams of solution (9) at the different values of parameter $a$ : $a=(1) 1$, (2) 10, (3) 50, and (4) 100 (see [21]). 
The case of $a<1$ corresponds to the negative surface energy (in Fig. 1a, this case is shown for $a=0.5$ ); it is not discussed in detail in this paper, because it is associated with the condensed phase instability.

The change in the free energy from the particle center to the particle surface can be estimated. This estimation allows the physical interpretation of the introduced parameters of the model and their comparison with conventional energy characteristics used to describe the nucleation process.

Let us initially consider the layer-by-layer change in this free energy of a cylindrical drop. Let us return to the formula for the energy that was used to derive the equation of motion. It is as follows: $E(\mathrm{r})=T+U$. Taking into account solution (9), we find that the kinetic energy is equal to the potential energy: $T=U$. This important result for the closed dynamic system is associated with the virial theorem for the finite motion; in our case, it is the test to verify whether this approach to the problem solution is correct. For the total full energy we have:

$$
E(\rho)=T+U=4 A\left(\frac{a}{\rho}\right)^{2} \frac{\rho^{2 a}}{\left(1+\rho^{2 a}\right)^{2}}
$$

It follows from (10) that, at $a=1$ and $r<1$, the equality $E(r)=A$ is satisfied. If $B>0$, then the cylinder surface energy tends to $A a^{2} \sim B$; the higher the $B$ value, the sharper the limit. Thus, just this parameter $B$ can be associated with the parameter of the specific thermodynamic surface energy that occurs in the thermodynamic theories (Gibbs, Tolman, etc.) provided that the dimension of these energies is different.

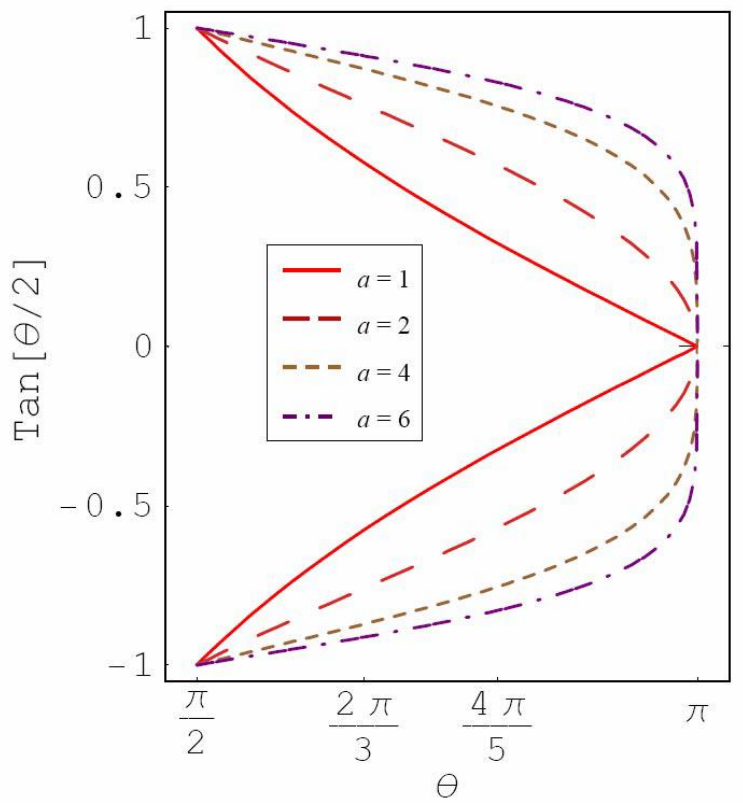

Fig. 2a. Plot for $\tan (\theta / 2)$ as function of $\theta$ for different $a$ values. 


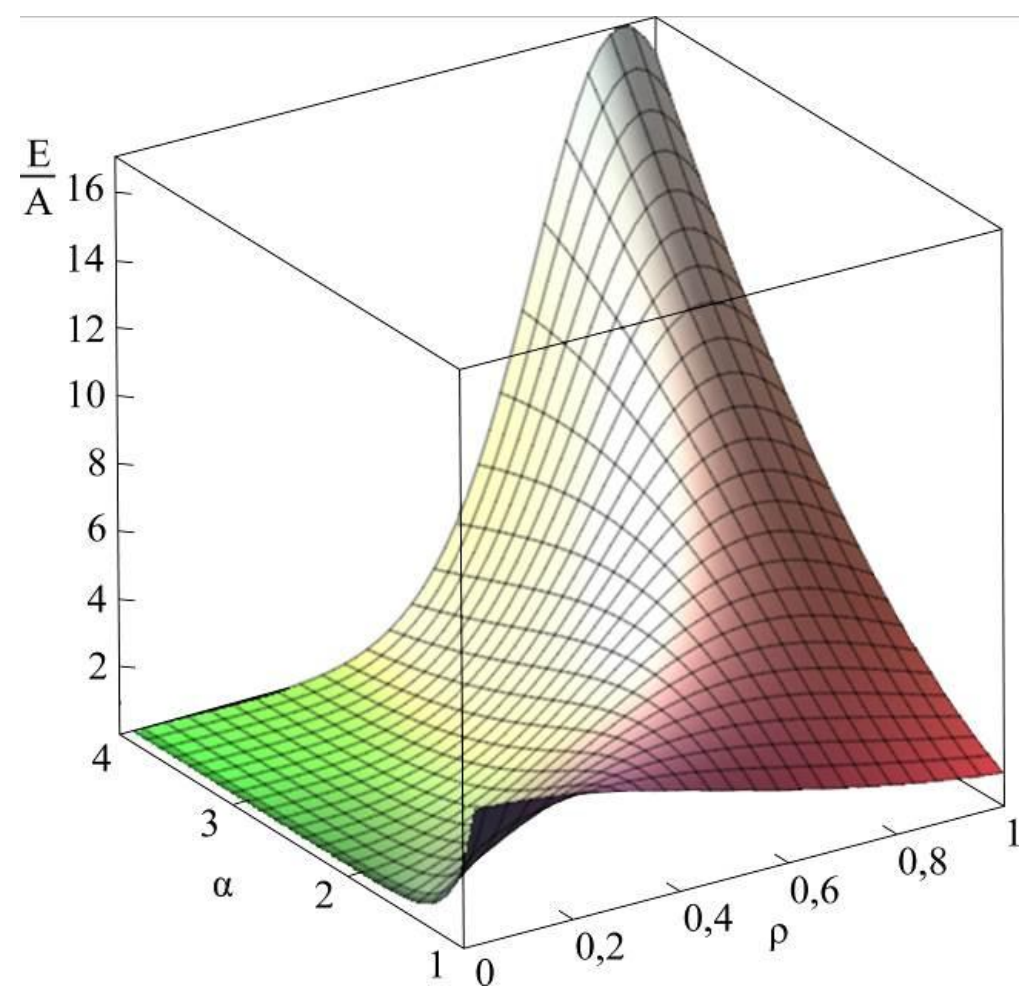

Fig. 2b. Dependence of energy on parameters $a$ and $\rho$ (see [22]).

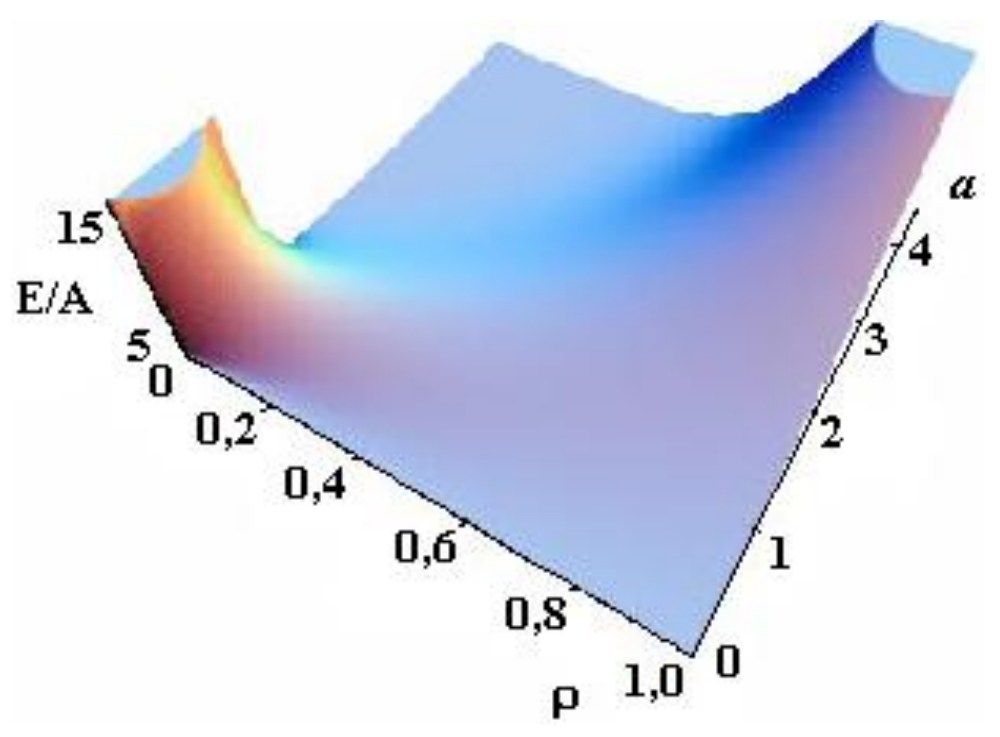

Fig. 2c. Dependence of energy on parameters $a$ and $\rho$ (see [21]).

A sharp rise in free energy (see Figs. 2b, 2c) depending on parameter $a$ is identified with 
the phase transition, which takes place in the system in the case of infinitely small anisotropy (for details see [21, 22]). To determine the total energy of the particle assigned to the cylinder length unit, an integral of $E(r)$ over the cylinder volume should be taken. Let us begin with a qualitative analysis of the model. Note that, for a particular case of $a=1$ and $B=0$, this integral should be equal to $A$ (with an accuracy to the multiplier). In this case, there is no other energy in the system; here $A$ is the only internal model energy of the system. In another limiting case, a certain high value of $a$ is sufficient for the total energy to tend to the anisotropy energy $B$. In the general case, the total full specific energy (for the cylinder length unit) will be as follows:

$$
W=2 \pi \int_{0}^{1} E(\rho) \rho d \rho=8 \pi a^{2} A \int_{0}^{1} \frac{\rho^{2 a-1} d \rho}{\left(1+\rho^{2 a}\right)^{2}}=2 \pi a A .
$$

In the Cahn-Hilliard theory [22, 23], the activation barrier energy is in proportion to the geometric mean of two energy parameters:

$$
E_{c} \sim \sqrt{A B}
$$

Unlike the proposed theory, the Cahn-Hilliard theory is not scale-invariant, and the quantity $B$ has a dimension of $\mathrm{J} / \mathrm{m}^{3}$. In our case, the integral formula derived from (11) for the activation energy has the same form; that is, the coincidence of these theories can be stated in calculating the mean activation energy (in the volume unit). Thus, we can deduce that the proposed theory qualitatively coincide with the Cahn-Hilliard theory. In terms of the Cahn-Hilliard theory, we obtain the same analytic structures [23]:

$$
\operatorname{tg} \theta(\rho) / 2=\exp \left[ \pm\left(\rho-\rho_{0}\right) / r_{c c}\right]
$$

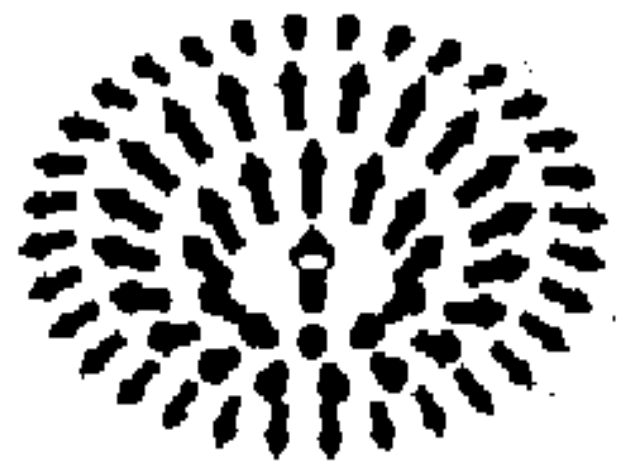

(A)

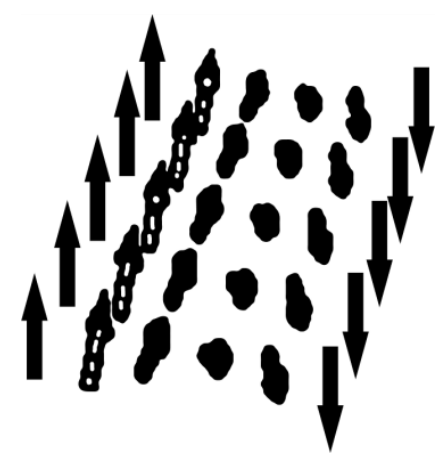

(B)

Fig. 3. Schematics representations of solution (9): (A) and (12): (B) in the form of a domain wall of energy vectors. 


\section{Physical Application}

The previously introduced parameter $a^{2}$ (see (7)) can formally be less than $a=1$ (in Fig. 1a, this case is shown for $a=0.5$ ). This case can correspond to negative anisotropy energy, which can, for example, prevent the formation of a nanoparticle. Technologically, it is possible to initiate the formation of a nanoparticle, yet limit the nanoparticle growth.

Therefore, it is of interest to consider the case where

$$
B=-A
$$

This physical situation can take place in the case when a nanoparticle nucleus with a size of $\rho_{0}<1$ (for definiteness, $\rho_{0}=0.1$ ) has already been formed; however, the development of it to an equilibrium state with $\rho=1$ is hindered by the created (artificially) anisotropy:

$$
\frac{-A \sin ^{2} \theta_{a}}{\rho^{2}} .
$$

We believe that this anisotropy manifests itself only starting from some sizes corresponding to the value

$$
\rho_{0}=0.1
$$

Then, for this case, the equation is greatly simplified:

$$
\theta^{\prime \prime}(\rho)+\frac{\theta^{\prime}(\rho)}{\rho}=0
$$

A particular solution for equation (16) can have the form (in our case, we use condition (16) to compare it with the solution in the form of formula (9)):

$$
\pi-\theta=c \ln \left(\rho / \rho_{0}\right)
$$

where

$$
c=\pi /\left(2 \ln \left(1 / \rho_{0}\right)\right), \quad\left(\text { for } \rho>\rho_{0} .\right) .
$$

Function graph $\quad c \ln \left(\rho / \rho_{0}\right) \quad$ is shown in Fig. 4. 

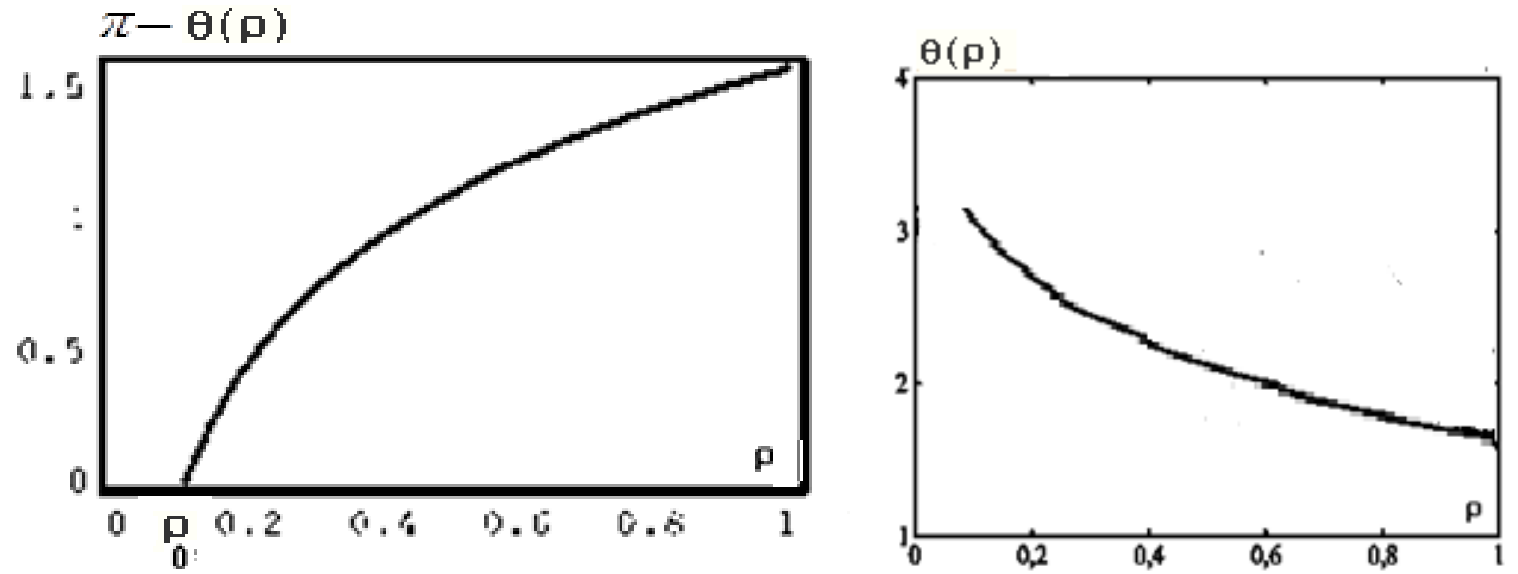

Fig. 4. Functions graphs of solution (17).

\section{Electrochemical Application}

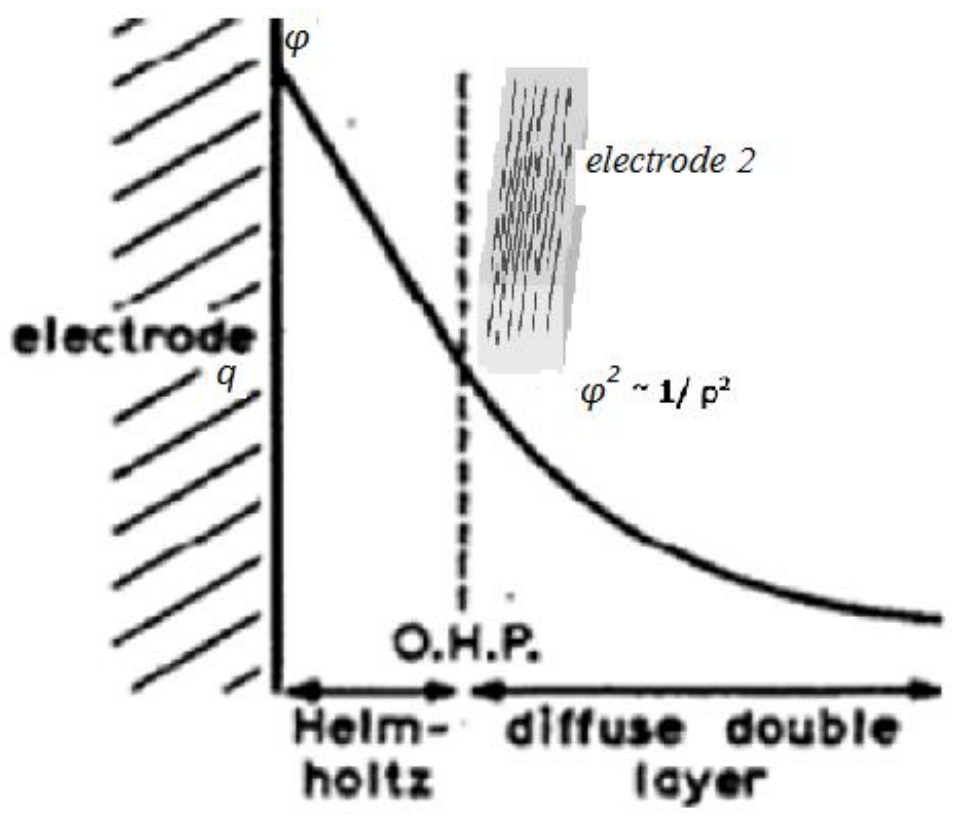

Fig. 5. Scheme of the physical process of nucleation for the case of electrochemical nucleation. The charge is $q$, the potential is $\varphi$. The capacity of the double layer is $C$. Electrode 2 (grid) can control the electrodeposition by changing the potential and electrical capacity of the near-electrode layer.

We can study the electrochemical nature of the examined anisotropy for the case of 
electrochemical nucleation. This anisotropy can be generated using the distribution of the electric field in the near-electrode layer, because the dimensions of the particles become comparable to the dimensions of the layer.

Let the surface energy change $d \sigma$ in the nucleation process according to the Lippman equation [25]:

$$
-d \sigma=q d \varphi
$$

where $q$ and $\varphi$ are the charge and potential on the surface. In the approximation of the constant capacity of the double layer $C$

$$
q=C \varphi
$$

Note that constant capacity $C$ is defined as a specific amount, namely, capacitance per unit area as surface energy $\sigma$ is defined as the amount of energy per unit area.

For the change in surface energy $\sigma$, we obtain

$$
\sigma=C(\varphi)^{2} / 2
$$

The change in the surface energy can be attributed to the anisotropy introduced above. Actually, if we assume that an asymptotic functional dependence occurs,

$$
(\varphi)^{2} \sim 1 / \rho^{2}
$$

then we finally obtain for parameter $a^{2}$

$$
a^{2} / r_{c} \sim C / 2
$$

where $r_{c} \sim 10^{-6} \mathrm{~cm}$ is the equilibrium value of the nanoparticle dimension.

For the evaluation, we take the specific capacity of a mercury electrode that is known to be on the order of

$$
C / 2 \sim 10^{7}(1 / \mathrm{cm})(\mathrm{CGS}) .
$$

If we confine ourselves to the upper limit of the $\boldsymbol{r}_{\boldsymbol{c}}$ value, then, for the evaluation of dimensionless quantity $a^{2}$, we obtain

$$
a^{2} \sim 10
$$

In general, the consideration results are qualitative.

\section{Mathematical Application}

In terms of the classical theory [8-10, 22-24], we can obtain (see Fig. 6) 


$$
\tan \left(\frac{\theta}{2}\right) \sim \frac{H}{\mathrm{R}}
$$

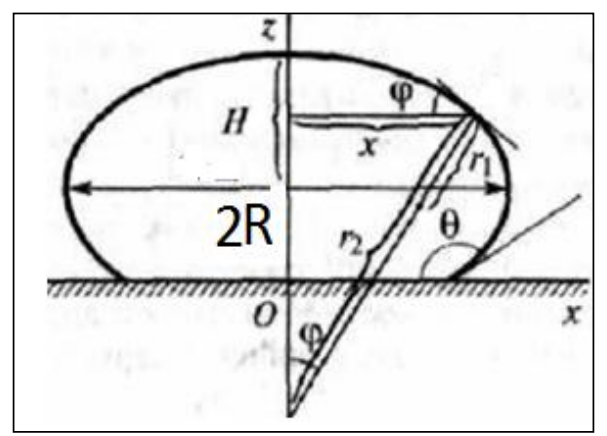

Fig. 6. Dimensionless profiles of a sessile microdrop.

From formulas (5)-(9), we can obtain the dependence

$$
\begin{aligned}
& \tan \left(\frac{\theta}{2}\right)=\frac{1}{\rho} \quad \text { and } \\
& \tan \left(\frac{\theta_{a}}{2}\right)=\frac{1}{\rho^{a}} .
\end{aligned}
$$

These formulas (18) and (5)-(9) have the same analytic structures.

In terms of the Cahn-Hilliard theory, we obtain same analytic structures (12). Another dependence, which can correspond to (5)-(9), is obtained in [26].

\section{Conclusions}

(i) We have obtained results associated with the van der Waals gradient theory, which can be resumed in the following way. If in the formation of a nanoparticle there is only one energy form that plays the role of exchange interaction $A$, then, in the context of the proposed model, the additive separation of the system energy into the surface energy and the nanoparticle volume energy is incorrect. However, in this case, we can introduce the average energy of the whole nanoparticle and, from simple geometric considerations, derive the Rusanov linear formula for the surface energy (see [22]). Typically, the Rusanov formula is assumed universally applicable. This fact is not confirmed when our model of the anisotropy energy is complicated.

(ii) The concept of anisotropy energy, which is introduced into the theory in the form of the proposed model as a modified Rapini potential, leads to the appearance of surface energy. Note that, in the conventional Rapini potential, there is no multiplier of the form of $1 / r^{2}[21,22]$. Anisotropy energy can have the meaning of double electric layer energy (in electrochemistry); in addition, in the case of the formation of extremely small equilibrium particles with a differentiated surface energy, the electric capacity of the nanosystem where this nanoparticle is 
formed should be increased. Thus, it can be assumed that the nano-nucleation process can be efficiently controlled.

Acknowledgments. This work was supported by the Moldavian national project and the Shevchenko Pridnestrov'e State University project.

\section{References}

[1] S. Ono and S. Kondo, Molecular Theory of Surface Tension in Liquids, in Structure of Liquids, series Encyclopedia of Physics, Springer, Berlin, Heidelberg, 1960, vol. 3/10, pp. 134-280.

[2] J. S. Rowlinson and B. Widom, Molecular Theory of Capillarity, Oxford University Press, Oxford, 1989.

[3] M. J. Jaycock and G. D. Parfitt, Chemistry of Interfaces, Halstead Press, John Wiley \& Sons, New York, 1981.

[4] A. W. Adamson and A. P. Gast, Physical Chemistry of Surfaces, Wiley-Interscience Publication, John Wiley \& Sons, 1997.

[5] A. I. Rusanov and V. A. Prokhorov, Interfacial Tensiometry, Elsevier, Amsterdam, 1996.

[6] R. C. J. Tolman, Chem. Phys. 17 (3), 333 (1949).

[7] S. Sh. Rekhviashvili and E. V. Kishtikova, Tech. Phys. 56 (1), 143 (2011).

[8] S. Sh. Rekhviashvili, Colloid J. 82(3), 342 (2020)

[9] A. A. Sokurov, Vestnik KRAUNC, Ser. Fiz.-Mat. Nauk 23 (3), 140 (2018).

[10] S. Sh. Rekhviashvili and A. A. Sokurov, Turk. J. Phys. 42, 699 (2018).

[11] T. V. Bykov and X. C. Zeng, J. Chem. Phys. 111 (23), 10602 (1999).

[12] T. V. Bykov and X. C. Zeng, J. Chem. Phys. 105 (47), 11586 (2001).

[13] I. A. Hadjiagapiou, J. Phys.: Condens. Matter 7, 547 (1995).

[14] V. I. Kalikmanov, J. Chem. Phys. 121, 8916 (2004).

[15] J. W. P. Schmelzer, G. Ropke, and V. B. Priezzhev, Nucleation Theory and Applications, JINR Publishing House, Dubna, 1999.

[16] J. W. P. Schmelzer, V. G. Baidakov, and G. Sh. Boltachev, J. Chem. Phys. 119, 6166 (2003).

[17] V. G. Baidakov and G. Sh. Boltachev, Phys. Rev. E 59 (1), 5648 (1997).

[18] V. G. Baidakov and G. Sh. Boltachev, Phys. Rev. E 59, 469 (1999).

[19] V. G. Baidakov, S. P. Protsenko, G. G. Chernykh, and G. Sh. Boltachev, Phys. Rev. E. 65, 047601 (2002).

[20] P. E. L'vov and V. V. Svetukhin, Phys. Stat. Sol. 57 (6), 1213 (2015).

[21] S. A. Baranov, in Handbook of Nanoelectrochemistry: Electrochemical Synthesis Methods, Properties and Characterization Techniques, Springer, International Publishing, Switzerland, 2015, pp. 1057-1069.

[22] S. A. Baranov, S. Sh. Rekhviashvili, and A. A. Sokurov, Surf. Eng. Appl. Electrochem. 55 (3), 286 (2019).

[23] S. A. Baranov, Surf. Eng. Appl. Electrochem., 53 (2), 124 (2017).

[24] S. A. Baranov, Mold. J. Phys. Sci. 19 (1-2), 182 (2020).

[25] P. Delahay, J. Chem. Educ. 43, 1, 54 (1966).

[26] A. Milchev and K. Binder, J. Chem. Phys. 117, 6852 (2018). 